\title{
Ruptured Pancreaticoduodenal Artery Pseudoaneurysm in an Unusual Location in a Patient with Chronic Pancreatitis: A Case Report
}

\author{
Sirote Wongwaisayawan ${ }^{1,2,0}$ Demetri Papadatos ${ }^{2}$ \\ ${ }^{1}$ Department of Diagnostic and Therapeutic Radiology, Faculty of \\ Medicine, Ramathibodi Hospital Mahidol University, Ratchathewi, \\ Bangkok, Thailand \\ 2Department of Medical Imaging, The Ottawa Hospital, Ottawa, \\ Ontario, Canada
}

Rima Aina ${ }^{2}$ Adnan Sheikh²

J Gastrointestinal Abdominal Radiol ISGAR

\begin{abstract}
Address for correspondence Sirote Wongwaisayawan, MD, Department of Diagnostic and Therapeutic Radiology, Faculty of Medicine, Ramathibodi Hospital Mahidol University, 270 Rama VI Road, Ratchathewi, Bangkok 10400, Thailand (e-mail: sirote.won@mahidol.edu).
\end{abstract}

\begin{abstract}
Keywords

- chronic pancreatitis

- pseudoaneurysm

- embolization

Bleeding pseudoaneurysm is a rare but yet potentially lethal complication in a patient with chronic pancreatitis. It also poses a diagnostic challenge among the radiologists and the attending physicians. The mortality rate can be as high as $50 \%$ depending on the clinical status, patient hemodynamics, site, and characteristics of the bleeding pseudoaneurysm. Multidisciplinary team approach is essential to deliver the optimum treatment strategy to each individual patient. In this article, we present a case of chronic pancreatitis that presents with acute epigastric pain caused by ruptured pancreaticoduodenal artery pseudoaneurysm in an unusual location. This patient was successfully treated with transcatheter coil embolization.
\end{abstract}

\section{Introduction}

Bleeding pseudoaneurysm in chronic pancreatitis is a rare vascular complication but potentially lethal. It is associated with a high mortality rate and poses a diagnostic challenge to the attending physicians. Imaging plays a significant role in the diagnosis of this serious condition.

\section{Case Presentation}

A 33-year-old male presented to our emergency department with acute onset of epigastric pain for 3 days. The patient had been diagnosed with chronic alcoholic pancreatitis for 5 years and diabetes mellitus for 1.5 years. The patient suffered from the last acute attack 7 months prior to this presentation. Magnetic resonance imaging performed at that time revealed a large pancreatic pseudocyst in the pancreatic body and tail with a maximal diameter of $6.7 \mathrm{~cm}$ ( - Fig. 1). The patient was treated conservatively and had been doing well since. Three days before admission, the patient had acute onset of epigastric pain and one episode of emesis. The patient also reported anorexia and was unable to eat.

published online October 12, 2020
DOI https://doi.org/ $10.1055 / \mathrm{s}-0040-1716804$ ISSN 2581-9933.
On admission, he had a blood pressure of 150/79 $\mathrm{mm} \mathrm{Hg}$, a regular heart rate of $60 \mathrm{bpm}$, and a body temperature of $36.3^{\circ} \mathrm{C}$. Clinical examination revealed moderate tenderness in the epigastric area. No rebound tenderness or signs of peritonitis were observed. The pain score was 6 out of 10 . The laboratory investigation revealed an elevated white blood cell count $\left(13.4 \times 10^{9} / \mathrm{L}\right)$ as well as elevated serum lipase level $(1,466 \mathrm{U} / \mathrm{L})$. His hemoglobin level was $113 \mathrm{~g} / \mathrm{L}$. The electrolyte panel and liver function tests were within normal limits. The international normalized ratio was 1.0.

An emergency contrast-enhanced computed tomography (CT) scan was obtained at the emergency department that showed multiple pancreatic calcifications as signs of chronic pancreatitis. No signs of acute pancreatitis were evident on the CT scan. However, there was an increase in size of the pancreatic pseudocyst to a maximum diameter of $8.6 \mathrm{~cm}$. The pseudocyst content appeared heterogeneously hyperdense ( $65-70$ Hounsfield unit), which represented internal hemorrhage. A $16-\mathrm{mm}$ pseudoaneurysm was visualized at the anterior aspect of the pseudocyst and was thought to originate from the left gastric or splenic artery at the time of interpretation (-Fig. 2). Interventional radiology consultation

(C)2020. Indian Society of Gastrointestinal and Abdominal Radiology. This is an open access article published by Thieme under the terms of the Creative Commons Attribution-NonDerivative-NonCommercial-License, permitting copying and reproduction so long as the original work is given appropriate credit. Contents may not be used for commercial purposes, or adapted, remixed, transformed or built upon. (https://creativecommons.org/licenses/by-nc-nd/4.0/). Thieme Medical and Scientific Publishers Pvt. Ltd. A-12, 2nd Floor, Sector 2, Noida-201301 UP, India 


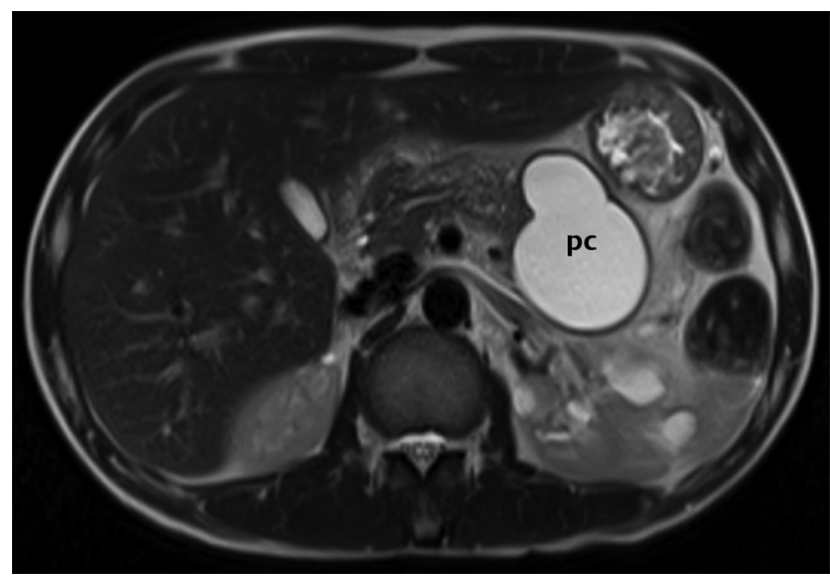

Fig. 1 Axial T2-weighted magnetic resonance image obtained 7 months prior to this presentation shows a T2-hyperintense pseudocyst (pc) at the pancreatic body and tail. The pseudocyst is clear without content.

was made after a discussion with the patient and a multidisciplinary care team. We decided to proceed with transcatheter embolization via the right femoral artery approach. Several angiograms in the different projections of the celiac artery, left gastric artery, superior mesenteric artery, splenic artery, and common hepatic artery failed to demonstrate active contrast extravasation or pseudoaneurysm. The dorsal pancreatic artery and transverse pancreatic artery were not demonstrated from the superselective angiograms. After a careful review of the vascular anatomy, superselective catheterization using a microcatheter into the gastroduodenal artery (GDA) was performed. This superselective angiogram revealed that there was a very short segment of GDA before giving off superior and inferior pancreaticoduodenal arteries. We advanced the microcatheter into the superior pancreaticoduodenal artery and eventually into the pancreaticoduodenal arcade. The pancreaticoduodenal arcade appears to end blindly with two small terminal vessels branched at its terminus. There was a communication from the blind end to the pseudoaneurysm ( $\boldsymbol{- \text { Fig. }}$ 3A). Two $2 \times 3 \mathrm{~cm}$ Nester coils (Cook Medical, Indiana, United States) were used for embolization, which resulted in complete occlusion of the feeding artery and no further opacification of the pseudoaneurysm ( $\sim$ Fig. 3B). After the coils were deployed, the microcatheter and the catheter system became dislodged proximally and ultimately we lost the vascular access. Because the patient's vital signs were stable during the procedure and the final angiograms demonstrated no further opacification of the pseudoaneurysm, we decided not to recannulate to deploy more coils. The follow-up CT angiography on the following day confirmed complete occlusion of the pseudoaneurysm. The patient tolerated the procedure well and was discharged from the hospital on the following day.

He had been doing well after 4 weeks of outpatient follow-up. He denied any vomiting, abdominal pain, melena, hematemesis, or anemic symptoms. Outpatient CT angiography had been scheduled. Unfortunately, the patient did not show up and lost to follow-up.

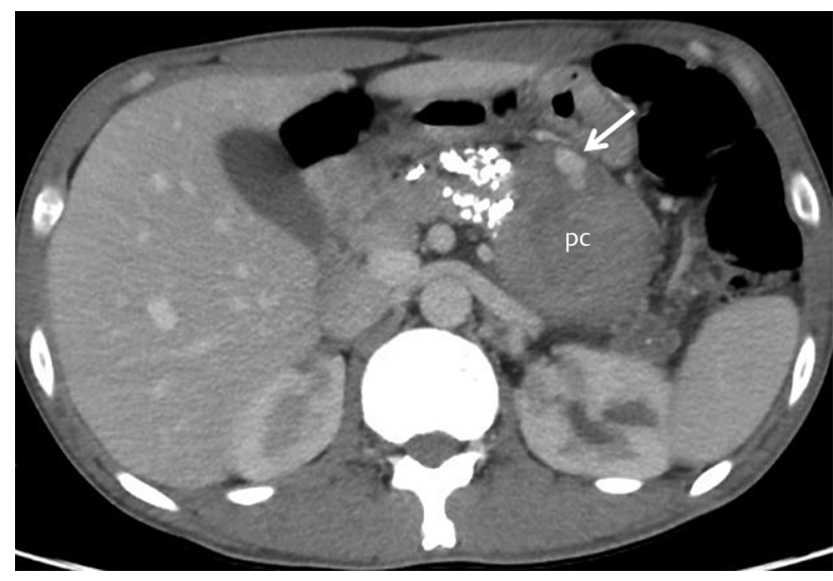

Fig. 2 Axial portovenous phase computed tomography image shows heterogeneous hyperattenuation of the pseudocyst (pc) and a pseudoaneurysm (arrow) at the anterior aspect.

\section{Discussion}

We herein described a rare complication of ruptured pancreaticoduodenal artery pseudoaneurysm in an unusual location in a patient who has chronic pancreatitis. He had been successfully treated with transcatheter coil embolization.

Chronic pancreatitis is an inflammatory condition that is usually irreversible and progressive. The vascular complications of chronic pancreatitis are the main causes of morbidity and mortality. Pseudoaneurysm formation after chronic pancreatitis is an uncommon complication with an estimated incidence of 10 to $17 \% .^{1,2}$ The formation of pancreatic pseudoaneurysm is thought to be secondary to long-standing erosion of the vessel wall by the pancreatic enzyme or due to the persistent extrinsic compression from the pseudocyst that eventually leads to ischemia and elastolytic degradation of the vessel wall. ${ }^{3}$ Splenic artery is the most common artery to be involved in almost half of the cases (30-50\%) due to close anatomical relationship to the pancreas as the splenic artery runs along posterior margin of the pancreas toward the splenic hilum. GDA and pancreaticoduodenal artery are the next commonly involved arteries ( $10-15 \%$ and $10 \%$ of the cases, respectively). ${ }^{4.5}$ The GDA typically arises from common hepatic artery and gives off anterior superior pancreaticoduodenal artery and posterior superior pancreaticoduodenal artery, forming pancreaticoduodenal arcades. These arteries and arcades serve as a major blood supply to the second part of duodenum and the pancreatic head. The GDA and pancreaticoduodenal artery pseudoaneurysm are usually found in the pancreatic head region as the GDA and the pancreaticoduodenal arcade lie in close proximity with the duodenal bulb and the pancreaticoduodenal groove. In this report, we described an unusual location of the pancreaticoduodenal artery pseudoaneurysm that originates from terminal branch of the pancreaticoduodenal arcade and locates in the pancreatic body.

Most of the patients who have pancreatic pseudoaneurysm usually remain asymptomatic. Unfortunately, when the pseudoaneurysm becomes symptomatic, the clinical 

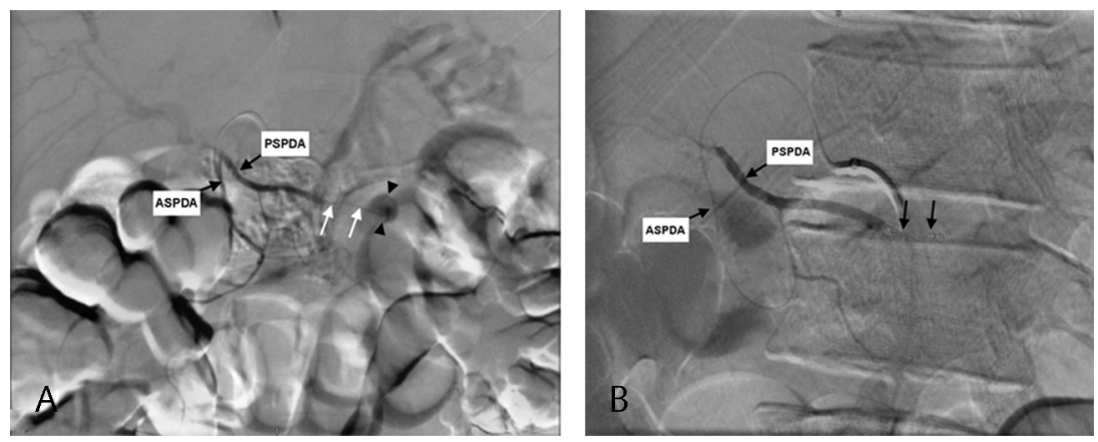

Fig. 3 Pre-embolization digital-subtraction angiogram of the pancreaticoduodenal arcade (A) shows blind-ended terminal vessel with a small communication (white arrow) to the pseudoaneurysm sac (arrowhead). Post-embolization image (B) shows coil embolization (arrow) and no opacification of the pseudoaneurysm sac. ASPDA, anterior superior pancreaticoduodenal artery; PSPDA, posterior superior pancreaticoduodenal artery.

presentation cannot be easily distinguished from an episode of acute pancreatitis, thus making a diagnosis very challenging. The pseudoaneurysm can rupture, and causes hemorrhage into the pancreatic pseudocyst, adjacent bowel lumen, or frankly into the intraperitoneal cavity, which can result in hemodynamic instability. About $3 \%$ of the patients with pseudoaneurysm develop hemorrhage and the mortality rate is as high as 30 to $50 \%$ if bleeding occurs. ${ }^{2,3,6,7}$ The risk of rupture is independent of the pseudoaneurysm size. ${ }^{8}$

Clinically, the possibility of bleeding pancreatic pseudoaneurysm should be considered if there are any of the following conditions: (1) sudden enlargement of a known pancreatic pseudocyst; (2) audible bruit over a pancreatic pseudocyst regardless of symptoms; (3) upper gastrointestinal bleeding in a patient with known pancreatic pseudocyst and absence of other cause seen on endoscopy; or (4) sudden drop in the hemoglobin level in a patient with known pancreatic pseudocyst, without other apparent cause. ${ }^{9}$ Unfortunately, the clinical presentation of acute abdominal pain in our patient is vague and cannot be distinguished from an episode of acute pancreatitis.

Imaging plays a significant role in the diagnosis of bleeding pancreatic pseudoaneurysm. Doppler ultrasound (US) can establish the diagnosis of pseudoaneurysm by demonstrating a classic "yin-yang sign" and "to-and-fro" spectral waveform..$^{3,79}$ However, US is operator dependent and has low sensitivity in detection of the pancreatic pseudoaneurysm due to its deep retroperitoneal location. ${ }^{10} \mathrm{CT}$ angiography has higher sensitivity and specificity in diagnosis of pancreatic pseudoaneurysm. The main advantages of CT angiography are wide availability, relatively fast acquisition, and less operator dependency. CT angiography also provides accurate size measurement, location of the pseudoaneurysm, and can evaluate pressure effect to the adjacent organs as well as the presence of active contrast extravasation. In addition, postprocessing with a three-dimensional reconstruction and maximum intensity projections can give important information for the treatment decision and preprocedural planning. However, the major drawbacks of CT are the radiation exposure, the need of intravenous contrast injection, and that concurrent interventional treatment is not possible.
Catheter angiography is accepted to be the most accurate investigation for diagnosis and treatment, but it is an invasive procedure and may not be available in all institutions. The major advantage of angiography is the ability to detect small pseudoaneurysm and the vessel of origin. Angiography can better define the location and character of the pseudoaneurysm and can serve both diagnostic and therapeutic purposes. ${ }^{11}$ Angiography is reported to have $100 \%$ sensitivity for the detection of arterial bleeding in patients with pancreatic pseudoaneurysms. ${ }^{3}$ The lesion that can mimic pancreatic pseudoaneurysm on other imaging modalities such as true aneurysm, arteriovenous fistula, or vascular malformation can be accurately diagnosed with angiography. ${ }^{3}$

The management of ruptured pancreatic pseudoaneurysm remains a challenging clinical problem. The treatment goal is to control the bleeding by either endovascular therapy or surgery. The preferred choice of treatment has long been debated and some authors suggested that the operation and endovascular therapy play complementary roles. ${ }^{12}$ Multiple factors such as hemodynamic status, comorbidities, and character of the bleeding pseudoaneurysm influence the treatment decision. Generally, a less invasive endovascular therapy is preferred to open surgery as it allows precise localization and characterization of the pseudoaneurysm, and assessment of the collateral vessels. ${ }^{13}$ Endovascular therapy also offers advantages in terms of less postoperative pain, shorter hospital length of stay, and early return to daily life activity. ${ }^{5}$ Embolization may also be a second option following unsuccessful surgery, complication of surgical procedure, or in patients with rebleeding after surgery. The currently available options for endovascular therapy include transcatheter embolization using coils, liquid embolic agents, or plugs; placement of a stent-graft; percutaneous thrombin injection; and combined or multimodal approach. ${ }^{13}$ The selection of the endovascular technique mainly depends on the morphological appearance and location of the pseudoaneurysm. The success rate of endovascular therapy is very high $(79-100 \%)$ with a low recurrent bleeding rate $(11-37 \%) .^{14-16}$ It is important to use Sandwich technique and to do both proximal and distal embolization for the GDA and pancreaticoduodenal artery pseudoaneurysm as well as most of the pseudoaneurysms of the gastrointestinal tract to 
prevent retrograde filling of the pseudoaneurysm sac from the rich collateral vessels. ${ }^{3}$ Unfortunately, in our case, the catheter system became dislodged proximally after we deployed the coils and ultimately the vascular access was lost. We decided not to recannulate and deploy more coils because of the fact that the pseudoaneurysm was communicated to blind end terminal vessel of pancreaticoduodenal artery and the final angiograms demonstrated no further opacification of the aneurysm.

Surgical treatment of pancreatic pseudoaneurysm can be challenging and is associated with higher morbidity and mortality. The surgery is usually performed either by pancreatectomy along with the ruptured pseudoaneurysm or direct ligation of the bleeding vessel. ${ }^{13,17}$ Surgery is usually reserved for hemodynamically unstable patients, unavailable or unsuccessful embolization, recurrent pseudoaneurysm after successful embolization, patients with infected pancreatic necrosis, or patients with the pseudoaneurysm located in the pancreatic head. ${ }^{18,19}$ Distal pancreatectomy and splenectomy may be an appropriate treatment for the bleeding pseudoaneurysm in the pancreatic tail because the endovascular treatment can be technically difficult and these surgical procedures are associated with low morbidity and mortality rates. ${ }^{14}$

\section{Conclusion}

Pseudoaneurysm formation with a contained hematoma in chronic pancreatitis is a rare but potentially fatal complication. Here we reported an unusual location of a ruptured pancreaticoduodenal artery pseudoaneurysm in the pancreatic body. Pseudoaneurysm rupture can occur at any time and can lead to hemodynamic instability. Early recognition of this complication is essential to prevent catastrophic consequences. Multidisciplinary team approach among the emergency radiologists, interventional radiologists, gastroenterologists, and surgical colleagues is ideal so that the optimum treatment strategy can be delivered to the patient.

\section{Authors' Contributions}

S.W. helped in idea and conceptualization, literature review, article draft, critical revision of article, and final approval for submission. D.P., R.A., and A.S. assisted in idea and conceptualization, critical revision of article, and final approval for submission.

\section{Funding}

None.

\section{Conflict of Interest}

None declared.

\section{References}

1 Volpi MA, Voliovici E, Pinato F, et al. Pseudoaneurysm of the gastroduodenal artery secondary to chronic pancreatitis. Ann Vasc Surg 2010;24(8):1136.e7-1136.e11
2 De Rango P, Parlani G, Silvestri V. Pseudoaneurysm in chronic pancreatitis. CMAJ 2012;184(4):433

3 Barge JU, Lopera JE. Vascular complications of pancreatitis: role of interventional therapy. Korean J Radiol 2012;13(Suppl 1):S45-S55

4 White AF, Baum S, Buranasiri S. Aneurysms secondary to pancreatitis. AJR Am J Roentgenol 1976;127(3):393-396

5 Gurala D, Polavarapu AD, Idiculla PS, Daoud M, Gumaste V. Pancreatic pseudoaneurysm from a gastroduodenal artery. Case Rep Gastroenterol 2019;13(3):450-455

6 Balthazar EJ, Fisher LA. Hemorrhagic complications of pancreatitis: radiologic evaluation with emphasis on $\mathrm{CT}$ imaging. Pancreatology 2001;1(4):306-313

7 Phillip V, Braren R, Lukas N, Schmid RM, Geisler F. Arterial pseudoaneurysm within a pancreatic pseudocyst. Case Rep Gastroenterol 2018;12(2):513-518

8 Habib N, Hassan S, Abdou R, et al. Gastroduodenal artery aneurysm, diagnosis, clinical presentation and management: a concise review. Ann Surg Innov Res 2013;7(1):4

9 Ryu K, Hong SS, Cha H, et al. A pancreatic hemorrhagic pseudocyst with pseudoaneurysm and the role of Doppler ultrasonography: a case report. Rev Assoc Med Bras (1992) 2019;65(2):123-126

10 Saad NEA, Saad WEA, Davies MG, Waldman DL, Fultz PJ, Rubens DJ. Pseudoaneurysms and the role of minimally invasive techniques in their management. Radiographics 2005;25(1, Suppl 1):S173-S189

11 Pérez C, Llauger J, Pallardó Y, Sanchís E, Sabaté JM. Radiologic diagnosis of pseudoaneurysms complicating pancreatitis. Eur J Radiol 1993;16(2):102-106

12 Carr JA, Cho JS, Shepard AD, Nypaver TJ, Reddy DJ. Visceral pseudoaneurysms due to pancreatic pseudocysts: rare but lethal complications of pancreatitis. J Vasc Surg 2000;32(4):722-730

13 Venturini M, Marra P, Colombo M, et al. Endovascular treatment of visceral artery aneurysms and pseudoaneurysms in 100 patients: covered stenting vs transcatheter embolization. J Endovasc Ther 2017;24(5):709-717

14 Hsu JT, Yeh CN, Hung CF, et al. Management and outcome of bleeding pseudoaneurysm associated with chronic pancreatitis. BMC Gastroenterol 2006;6(1):3

15 Zyromski NJ, Vieira C, Stecker M, et al. Improved outcomes in postoperative and pancreatitis-related visceral pseudoaneurysms. J Gastrointest Surg 2007;11(1):50-55

16 Bergert H, Dobrowolski F, Caffier S, Bloomenthal A, Hinterseher I, Saeger HD. Prevalence and treatment of bleeding complications in chronic pancreatitis. Langenbecks Arch Surg 2004;389(6):504-510

17 Larrey Ruiz L, Luján Sanchis M, Peño Muñoz L, et al. Pseudoaneurysm associated with complicated pancreatic pseudocysts. Rev Esp Enferm Dig 2016;108(9):583-585

18 Manazer JR, Monzon JR, Dietz PA, Moglia R, Gold M. Treatment of pancreatic pseudoaneurysm with percutaneous transabdominal thrombin injection. J Vasc Surg 2003;38(3):600-602

19 Udd $\mathrm{M}$, Leppäniemi AK, Bidel S, Keto $\mathrm{P}$, Roth WD, Haapiainen RK. Treatment of bleeding pseudoaneurysms in patients with chronic pancreatitis. World J Surg 2007;31(3):504-510 